\title{
Comparing Non-invasive Diagnostic Methods for Arteriovenous Fistula Stenosis: A Prospective Study
}

\section{Sukit Raksasuk}

Mahidol University Faculty of Medicine Siriraj Hospital

\section{Satit Rojwatcharapibarn}

Mahidol University

Thatsaphan Srithongkul ( $\nabla$ thatsaphan.sri@mahidol.ac.th )

Mahidol University Faculty of Medicine Siriraj Hospital https://orcid.org/0000-0002-5860-3395

Research article

Keywords: hemodialysis, arteriovenous fistula, arteriovenous fistula stenosis, access flow

Posted Date: July 28th, 2020

DOI: https://doi.org/10.21203/rs.3.rs-18618/v2

License: (a) (i) This work is licensed under a Creative Commons Attribution 4.0 International License.

Read Full License 


\section{Abstract}

Background International guidelines recommended screening for AVF stenosis using various non-invasive methods. The previous studies have reported conflicting results. This study aims to evaluate the utility of the different non-invasive AVF flow measurements for detecting AVF stenosis. Methods We evaluated 23 HD patients with the clinical suspicion for AVF stenosis based on physical examination or high venous pressures during. Patients underwent all three non-invasive measurements including ultrasound dilution, urea dilution, and Doppler ultrasonography. Fistulography was performed to confirm the degree of AVF stenosis in all patients. Results Fistulography revealed AVF stenosis in eighteen patients, twelve of whom had severe stenosis (greater than $50 \%$ stenosis). About the location of the stenotic lesions, eight were at the inflow site, six at the outflow site, and four at both sites. In those AVFs with severe stenosis, median access flows were $625 \mathrm{~mL} / \mathrm{min}$ by ultrasound dilution method, $615 \mathrm{~mL} / \mathrm{min}$ by urea dilution method, and $590 \mathrm{~mL} / \mathrm{min}$ by Doppler ultrasonography. Receiver operating characteristic (ROC) curve analysis showed that Doppler ultrasonography had a high discriminative ability and the averaged areas under the curves were 0.933 ( $95 \%$ confidence interval [Cl], 0.81 to 0.99 ) for stenosis and $0.929(95 \% \mathrm{Cl}, 0.82$ to 0.99$)$ for severe stenosis. The sensitivity of each method for prediction of access stenosis was $73 \%, 73 \%, 80 \%$ by using the ultrasound dilution, urea dilution, and Doppler ultrasonography methods, respectively. The specificity of each method was $40 \%, 80 \%$, and $100 \%$ respectively. Physical examination showed $80 \%$ sensitivity and $80 \%$ specificity for detecting AVF stenosis. The combination of non-invasive access flow measurement with physical examination increased the sensitivity for detection of AVF stenosis to $80 \%$, $93 \%$, and $93 \%$ respectively. Conclusions Doppler ultrasound combine with physical examination, was more accurate than other non-invasive methods for detection of AVF stenosis.

\section{Background}

Reliable, arterio-venous access is of critical importance for patients with end-stage renal disease (ESRD) on hemodialysis (HD). An arteriovenous fistula (AVF) is the ideal hemodialysis vascular access, because of a lower infection rate and associated morbidity and mortality as compared to either arteriovenous graft (AVG) or central venous catheter (CVC) [1, 2] . However, long term AVF patency is a significant problem; recently studies revealed one-year AVF survival of 40-90\% [3-6]. AVF stenosis from neointimal hyperplasia leading to thrombosis in any segment of the fistula is the most common cause of loss of patency $[5,7,8]$. Early manifestations from the physical examination include weak fistula, decreased arterial or increased venous pressure, limb edema, loss of AVF collapse after arm elevation, and decreased thrill in AVF[5, 9, 10].

Therefore, European Society of Vascular Surgery (ESVS) Guidelines 2018 recommend surveillance of the vascular access by non-invasive methods to identify stenotic lesions early so that interventions can be organized and the risk of later thrombosis mitigated $[10,11]$. Otherwise, the Kidney Disease Outcomes Quality Initiative (KDOQI) guideline 2019 did not recommend doing routine AVF surveillance by measuring access blood flow, pressure monitoring, or imaging for stenosis for additional to routine clinical monitoring.[12] 
Fistulography is the gold standard method for diagnosis of AVF stenosis, but this is an invasive method that incurs high costs, involves contrast exposure, and is not available in some HD centers. Currently, non-invasive access flow measurements have been proposed, including ultrasound dilution technique, urea dilution, and color Doppler ultrasonography $[10,13,14]$.

There is no substantial evidence to guide the choice of non-invasive surveillance technique. The screening algorithm of $\mathrm{AV}$ access flow by ultrasound dilution resulted high sensitivity for detecting hemodynamically significant stenosis [15].

Urea recirculation, which calculated access flow from inducing urea recirculation by reversing bloodline had a high sensitivity and specificity in detecting needed for intervention. However, this method works well for low blood flow, whereas insensitive at high flow $[13,16]$ Additionally, ultrasound dilution and urea circulation methods are strongly related to blood pressure and it might be better to calculate a ratio between the measured access flow and blood pressure.

Color Doppler ultrasonography can evaluate the access flow and identify the stenosis sites in the AVF, which is not possible with the urea and dilation technique[17]. However, this technique is operator dependent and time-consuming. Several studies have shown the benefit of noninvasive monitoring tools for detecting vascular access stenosis [13, 18-21]. However, none of the studies have compared three noninvasive tools: urea dilution, ultrasound dilution, and Doppler ultrasound) for detecting patients with clinical suspicion for AVF stenosis. In this study, we aimed to evaluate the utility of the different noninvasive access flow measurement methods (urea dilution, ultrasound dilution, and Doppler ultrasonography) for detecting AVF stenosis in patients on HD with a mature AVF and clinical suspicion of AVF stenosis.

\section{Methods}

\section{Study Design and Patients}

This cross-sectional study was conducted in patients with native mature AVFs receiving HD at the Siriraj Hospital Medical School, Bangkok, Thailand during January December 2018. The definition for mature AVF was defined by diameter at least $6 \mathrm{~mm}$ and blood flow rate reached $600 \mathrm{ml} / \mathrm{min}$. The study was performed in accordance with the Declaration of Helsinki and Good Clinical Practice guidelines. Ethics committees and institutional review boards approved the research protocol. All patients gave written informed consent before entry.

Physical examination (PE) was performed in 115 patients receiving HD during January - December 2018 in our dialysis center by one hemodialysis staff nurse and two 
nephrologists. The inter-observer variability of physical examination was defined as $5 \%$ among staff nurse and nephrologist. We recruited the patients who had features of AVF stenosis, including limb edema, loss of AVF collapse after arm elevation, high arterial negative pressure, prolonged bleeding from puncture site after needle withdrawal or high venous pressures of over $200 \mathrm{mmHg}$ on three consecutive HD runs. The other dialysis parameters were also reviewed for additional evidence (i.e., unexplained hemodialysis inadequacy, difficulty needling). Those patients with suspicion for AVF stenosis underwent three non-invasive tests looking for objective evidence of stenosis, as well as fistulography (Figure 1). Antegrade puncture with an 18G needle at dialysis fistula was performed and venography was done to evaluate patency of dialysis fistula and venous out flow as well as central veins. Retrograde access with a $5 \mathrm{Fr}$ vascular sheath at dialysis fistula and a $5 \mathrm{Fr}$ catheter was used to retrogradely select pass through anastomosis to artery. Then angiography was performed to evaluate artery, arteriovenous anastomosis and juxtaanastomosis. Inflow stenosis was defined as any stenosis within the arterial system, arteryvein anastomosis, and juxta-anastomosis region up to the arterial site or $2 \mathrm{~cm}$ downstream from the arterial anastomosis. Outflow stenosis was defined as any stenosis located beyond the cannulation area downstream from the venous needle and the locations up to the atrium[22]. The presence of a significant stenosis (severe) was defined by a $50 \%$ reduction in vessel diameter compared with the adjacent segment.

\section{AVF Assessment}

The protocol for investigation was as follows: each patient with clinical suspicion for AVF stenosis underwent dilution ultrasound, urea dilution studies, and Doppler ultrasound scans in first, second and third week, respectively. Each test was repeated twice time in consecutive hemodialysis sessions. During transonic ultrasound and urea dilution studies were performed in the first hour of dialysis with the same blood flow rate in all patients. All patients underwent fistulography within four weeks of completion of the non-invasive tests.

The ultrasound dilution technique utilized the Transonic $^{\circledR}$ HD Monitor. The measurement was taken twice within 30 - 60 minutes after HD initiation. Ultrafiltration was 
turned off during measurement in all patients. The urea dilution method test was performed within 30 - 60 minutes of starting HD by the same hemodialysis nurse. After line reversal, arterial (a) and venous (v) samples were drawn at a blood flow rate (Qb) of $350-400$ $\mathrm{mL} / \mathrm{min}$, and the systemic (s) sample was drawn from the arterial tubing after the blood pump was stopped. Blood urea nitrogen (BUN) concentrations were measured in all samples. The recirculation (R) and access flow (Qa) were calculated as follow:

$$
\begin{aligned}
& \mathrm{R}=100 \times \frac{(\mathrm{BUN} s-\mathrm{BUNa})}{\mathrm{BUN} s-\mathrm{BUNV})} \\
& \mathrm{Q} \mathrm{a}=\mathrm{Qb} \times \frac{(1-\mathrm{R})}{\mathrm{R}}
\end{aligned}
$$

Nephrologist and Radiologist independently performed color Doppler ultrasonography in the same day for each patient. The results of access flow from all techniques were calculatec and presented as mean value and median for symmetrical and asymmetrical dato distribution; respectively. A7-15 MHz linear transducer was used. Peak systolic velocity anc AVF diameter were measured at the anastomosis, juxta-anastomosis, body, and outflow site of the AVF. Access flow (Qa) was calculated as follow:

Access flow $(\mathrm{mL} / \mathrm{min})=$ Time-averaged mean velocity $(\mathrm{cm} / \mathrm{sec}) \times \Pi \times$ radius $^{2} \times 60(\mathrm{sec})-$ (3)

The Access flow measurement was performed based on the standardize protocol using a Toshiba Xario 100 and a PLU-704BT linear transducer with patients in a supine position. Waveforms of the arterial inflow and the arterial anastomosis were obtained at over $10 \mathrm{~cm}$ of brachial artery, which demonstrated by Transverse and longitudinal B-mode and color flow images. The access flow was done twice times for each patient and calculated for the average access flow.[23]

Fistulography was performed by the interventional radiologist to evaluate the vascular access from the feeding artery to the vein. The AV fistula with stenosis more than 50 percent was defined as significant stenosis and may require angioplasty.

\section{Statistical Analysis}


Data are presented as percentages, mean \pm standard deviations (SD), or medians with $95 \%$ confidence interval [CI]. Receiver-operating characteristic (ROC) curves were analyzed to determine the accuracy of the three non-invasive tests, as measured by the area under the curve (AUC), and to identify optimal cutoffs for continuous variables. P values less than 0.05 were considered statistically significant. SPSS software, version 20, was used for analysis.

\section{Results}

One hundred and fifteen HD patients provided written inform consent. 87 patients were excluded due to i) did not meet the criteria for AVF stenosis $(n=39)$, ii) used AVG or CVC $(n=48)$. The remaining 28 patients completed all baseline assessments and were enrolled in the study. Five patients were excluded due to not completed all vascular access measurements. Therefore, 23 patients were enrolled in the final analysis.

(Figure 1)

Twenty-three patients, who had clinical suspicion for AVF stenosis, were recruited. The characteristics of the patients and their AVFs are summarized in Table 1. The median time of dialysis duration was 5.5 years. Fourteen (60\%) of the fistulas were located in the forearm (radiocephalic), and nine (40\%) were located in the upper arm (brachiocephalic). Fifteen fistulas (65\%) revealed physical signs suspicious for AVF stenosis. 'Thirty-five percent of the patients showed significant increased venous pressure during dialysis time without any anomaly at physical examination.

The median access flows were 805,828 , and $604 \mathrm{~mL} / \mathrm{min}$ by ultrasound dilution, urea dilution, and Doppler ultrasonography methods, respectively. Fistulography revealed AVF stenosis in eighteen patients, twelve of whom had significant stenosis (greater than $50 \%$ ). The locations of the stenosis lesions were eight at the juxa-anatomosis, six at body of venous segment, and four patients had stenosis at both juxta anastomosis and any part of venous segment. The median access flows in the non-significant stenosis group and the significant stenosis group were shown in Table 2. In significant stenosis group, the ROC showed an area under the curve (AUC) of access flow was $0.53(95 \% \mathrm{Cl}, 0.26$ to 0.85$)$ from ultrasound dilution, $0.63(95 \% \mathrm{Cl}, 0.32$ to 0.93$)$ for urea dilution, and $0.93(95 \% \mathrm{Cl}, 0.81$ to 1.00$)$ for Doppler ultrasound (Figure2). Doppler ultrasound is a highly accurate method for detection of non- significant AVF stenosis (less than $50 \%$ ) compared to the other non-invasive methods with statistical significance $(P<$ 0.01 for both ultrasound dilution and urea dilution techniques). Sensitivity and specificity for detection of non-significant AVF stenosis by Doppler ultrasound were $80 \%$ and $100 \%$ (Table 3 ).

At ROC curve analysis, the method with the best discriminatory capacity for diagnosis of significant AVF stenosis (more than $50 \%$ ) was Doppler ultrasound with an AUC of $0.93(95 \% \mathrm{Cl}, 0.82$ to 1.00$)$. The other methods demonstrated an AUC of $0.74(95 \% \mathrm{Cl}, 0.52$ to 0.97$)$ for ultrasound dilution, and $0.82(95 \% \mathrm{Cl}$, 0.63 to 1.00 ) for urea dilution in detecting significant stenosis. Sensitivity and specificity for the diagnosis of significant stenosis of AVF by Doppler ultrasound were $100 \%$, and $82 \%$, respectively. Sensitivity and specificity by other methods are shown in Table 4. 
In this study, physical examination had $80 \%$ sensitivity and $80 \%$ specificity for the diagnosis of AVF stenosis. Combining physical examination with non-invasive methods had improved their accuracy for diagnosis of AVF stenosis. The combinations of a positive physical examination with ultrasound dilution and urea dilution improved the sensitivity to $80 \%$ and $93 \%$, respectively.

There were no differences between the two raters (Nephrologist and Radiologist) in terms of access flow measurement by Doppler ultrasound, the corresponding coefficient of variation (CV \%) was 4.73, 4.53 respectively. However, for the dilution method, which was operated by hemodialysis staff nurses, we found $\mathrm{CV}$ equal to $8 \%$.

\section{Discussion}

This cross-sectional study evaluated the characteristics of the three non-invasive methods for the detection of AVF stenosis. We found that the Doppler ultrasound had the best accuracy in detecting and locating AVF stenosis comparing to other non-invasive tests. European Society of Vascular Surgery (ESVS) Guidelines recommended flow measurement of arteriovenous fistulas every 3 months for hemodynamically significant stenosis that can be treated to prevent thrombosis and prolong the longevity of the AVF.[11] This approach has been questioned with several issues concerning the predictive accuracy of the screening methods [20, 24-26]. The data from a systematic review found that prospective surveillance of AVFs for significant stenosis, when combined with correction of the anatomic stenosis, could decrease the incidence of thrombosis but was not significant in preventing access loss. [27, 28] However, to evaluate the AV fistula function several studies proposed noninvasive methods for early detection and early correction. Several studies evaluated bedside procedures for measurement of access flow. The previous study demonstrated that was no significant different in access flow measurement between ultrasound dilution method and duplex ultrasonography[29]. Furthermore, there was no different in access flow measurement accuracy of AV fistula and AV graft among ultrasound dilution compared with color Doppler ultrasound technique[30]. Unfortunately, it is still controversial which tool is better for evaluating and monitoring vascular access [21, 31-34]. In this study, we aimed to demonstrate the accuracy of various tools for detecting AVF stenosis in the patient with clinical suspicion for stenosis. Doppler ultrasound showed a high discriminatory capacity for diagnosis of AVF stenosis. That said, there are variability and operator dependence. We also assessed the inter-rater agreement for Doppler ultrasound, which found no significant difference between raters.

Regarding accuracy in diagnosing stenosis in AVF, the available studies have reported on both AV grafts and AVFs, in an uncontrolled, non-randomized fashion our results suggest Doppler ultrasound is a highly accurate method to detect AVF stenosis $[20,22,26,31]$. The sensitivity and specificity in this study were different from the previous study because the eligible patients in this study had clinical suspicion for AVF stenosis by physical examination and/or venous pressure more than $200 \mathrm{mmHg}$. In this study, we showed that physical examination is highly sensitive and specific for detecting AVF stenosis, in particular when combined with Doppler ultrasound. 
From our study, Doppler ultrasound is useful for detecting AV fistula stenosis. Physical examination combined with urea dilution may be an alternative for detecting AVF stenosis when Doppler ultrasound is not available. Several studies demonstrated a role of both urea and ultrasound dilution in the detection of recirculation and abnormal access flow $[14,21,28,33,35,36]$

Our study had some limitations. Firstly, it is single-center nature with small patient numbers. Moreover, a small number of negative result from fistulography may affect the specificity accuracy. Unfortunately, we included only symptomatic patients because the investigation was quite invasive, especially fistulography. However, the inclusion criteria for AV fistula stenosis in our study included high venous pressure, which was early sign for detecting access malfunction. Secondly, information regarding longterm AV fistula patency and survival was not available. Lastly, we did not demonstrate the secondary patency after AVF stenosis correction. The future study that included larger patient number may be need to demonstrate the accuracy of non-invasive method in detecting AVF stenosis.

\section{Conclusion}

In conclusion, access flow measurement by Doppler ultrasound is highly accurate for detecting AVF stenosis in patients with clinical suspicion for AV fistula stenosis. Doppler ultrasound combined with physical examination remains an essential tool for detecting AVF stenosis in patients on HD.

\section{Abbreviations}

AVF; Arteriovenous fistula, HD; Hemodialysis, ROC; Receiver operating characteristic, ESRD; End stage renal disease, Qb; blood flow rate, BUN; blood urea nitrogen, R; recirculation, Qa; Access flow, a; arterial, v; venous

\section{Declarations}

\section{Availability of data and materials}

The data generated and analyzed during the current study are available from the corresponding author on reasonable request.

\section{Acknowledgement}

Not applicable

\section{Funding}

No funding

\section{Competing interest}

None of the authors had any conflict of interest in relation to this study. 
Author information

\section{Affiliations}

${ }^{1}$ Nephrology Division, Department of Medicine, Faculty of Medicine, Siriraj Hospital, Mahidol University

${ }^{2}$ Department of Radiology, Faculty of Medicine, Siriraj Hospital, Mahidol University

\section{Author's Contributions}

SK and TS were involved in the study design. SK, SR and TS contributed to data collection. SK and TS completed analysis and writing of the manuscript. SK and TS contributed to data analysis and editing of the manuscript. All the authors participated in critical reading, commenting, and final acceptance of the article. The authors read and approved the final manuscript.

\section{Ethics declarations}

\section{Ethics approval and consent to participate}

Ethical approval was obtained from Siriraj Institutional Review Board, Faculty of Medicine, Siriraj hospital, Mahidol University (EC number 665/2560)

\section{Consent for publication}

Not applicable.

\section{References}

1. Lok CE, Huber TS, Lee T, Shenoy S, Yevzlin AS, Abreo K, et al. KDOQI clinical practice guideline for vascular access: 2019 update. American Journal of Kidney Diseases. 2020;75(4):S1-S164.

2. Pisoni RL, Zepel L, Port FK, Robinson BM. Trends in US vascular access use, patient preferences, and related practices: an update from the US DOPPS practice monitor with international comparisons. American Journal of Kidney Diseases. 2015;65(6):905-915.

3. Pisoni RL, Young EW, Dykstra DM, Greenwood RN, Hecking E, Gillespie B, et al. Vascular access use in Europe and the United States: results from the DOPPS. Kidney international. 2002;61(1):305-316.

4. Huijbregts HJ, Bots ML, Wittens $\mathrm{CH}$, Schrama YC, Moll FL, Blankestijn PJ. Hemodialysis arteriovenous fistula patency revisited: results of a prospective, multicenter initiative. Clinical Journal of the American Society of Nephrology. 2008;3(3):714-719.

5. Kazemzadeh G, Modaghegh M, Ravari H, Daliri M, Hoseini L, Nateghi M. Primary patency rate of native AV fistula: long term follow up. International journal of clinical and experimental medicine. 2012;5(2):173.

6. Jennings WC, Parker DE. Creating arteriovenous fistulas using surgeon-performed ultrasound. J Vasc Access. 2016;17(4):333-339. 
7. Roy-Chaudhury P, Spergel LM, Besarab A, Asif A, Ravani P. Biology of arteriovenous fistula failure. Journal of nephrology. 2007;20(B):150.

8. Lee T, Roy-Chaudhury P. Advances and new frontiers in the pathophysiology of venous neointimal hyperplasia and dialysis access stenosis. Advances in chronic kidney disease. 2009;16(5):329-338.

9. Beathard GA, Settle SM, Shields MW. Salvage of the nonfunctioning arteriovenous fistula. American Journal of Kidney Diseases. 1999;33(5):910-916.

10. Found NK. KDOQI clinical practice guidelines and clinical practice recommendations for 2006 updates: Hemodialysis adequacy, peritoneal dialysis adequacy and vascular access. Am J Kidney Dis. 2006;48(Suppl 1):S1-S322.

11. Schmidli J, Widmer MK, Basile C, de Donato G, Gallieni M, Gibbons CP, et al. Editor's Choice - Vascular Access: 2018 Clinical Practice Guidelines of the European Society for Vascular Surgery (ESVS). Eur J Vasc Endovasc Surg. 2018;55(6):757-818.

12. Allon M. Lessons From International Differences in Vascular Access Practices and Outcomes. American Journal of Kidney Diseases. 2018;71(4):452-454.

13. Koirala N, Anvari E, McLennan G, editors. Monitoring and surveillance of hemodialysis access. Seminars in interventional radiology; 2016: Thieme Medical Publishers.

14. Schwarz C, Mitterbauer C, Boczula M, Maca T, Funovics M, Heinze G, et al. Flow monitoring: performance characteristics of ultrasound dilution versus color Doppler ultrasound compared with fistulography. American Journal of Kidney Diseases. 2003;42(3):539-545.

15. Ashoor IF, Hughson EA, Somers MJG. Arteriovenous Access Monitoring with Ultrasound Dilution in a Pediatric Hemodialysis Unit. Blood Purification. 2015;39(1-3):93-98.

16. Vega A, Abad S, Aragoncillo I, Galan I, Macias N, Cedeno S, et al. Comparison of urea recirculation and thermodilution for monitoring of vascular access in patients undergoing hemodialysis. J Vasc Access. 2018;19(3):283-290.

17. Sands J. The role of color-flow Doppler ultrasound in the management of hemodialysis accesses. ASAIO journal. 1998;44(1):41-43.

18. Schwab SJ, Oliver MJ, Suhocki P, Mccann R. Hemodialysis arteriovenous access: detection of stenosis and response to treatment by vascular access blood flow. Kidney international. 2001;59(1):358-362.

19. Kleinekofort W. Method of monitoring a vascular access and an apparatus for extracorporeal treatment of blood with a device for monitoring the vascular access. Google Patents; 2003.

20. Lopot F, Nejedlý B, Válek M. Vascular Access Monitoring: Methods and Procedures-Something to Standardize? Blood Purification. 2005;23(1):36-44.

21. Segal JH, Weitzel WF. Monitoring techniques of vascular access. Contributions to nephrology. 2004;142:216-227.

22. Salman L, Ladino M, Alex M, Dhamija R, Merrill D, Lenz O, et al., editors. Accuracy of ultrasound in the detection of inflow stenosis of arteriovenous fistulae: results of a prospective study. Seminars in dialysis; 2010: Wiley Online Library. 
23. Back MR, Maynard M, Winkler A, Bandyk DF. Expected Flow Parameters Within Hemodialysis Access and Selection for Remedial Intervention of Nonmaturing Conduits. Vascular and Endovascular Surgery. 2008;42(2):150-158.

24. Krivitski NM, MacGibbon D, Gleed RD, Dobson A. Accuracy of dilution techniques for access flow measurement during hemodialysis. American Journal of Kidney Diseases. 1998;31(3):502-508.

25. Lindsay R, Bradfield E, Rothera C, Kianfar C, Malek P, Blake P. A comparison of methods for the measurement of hemodialysis access recirculation and access blood flow rate. ASAIO journal (American Society for Artificial Internal Organs: 1992). 1998;44(1):62-67.

26. Teodorescu V, Gustavson S, Schanzer H. Duplex ultrasound evaluation of hemodialysis access: a detailed protocol. International journal of nephrology. 2012;2012.

27. Polkinghorne KR, Lau KK, Saunder A, Atkins RC, Kerr PG. Does monthly native arteriovenous fistula blood-flow surveillance detect significant stenosis-a randomized controlled trial. Nephrology Dialysis Transplantation. 2006;21(9):2498-2506.

28. Tonelli M, James M, Wiebe N, Jindal K, Hemmelgarn B, Network AKD. Ultrasound monitoring to detect access stenosis in hemodialysis patients: a systematic review. American Journal of Kidney Diseases. 2008;51(4):630-640.

29. Sands J, Glidden D, Miranda C. Hemodialysis access flow measurement. Comparison of ultrasound dilution and duplex ultrasonography. Asaio j. 1996;42(5):M899-901.

30. Depner TA, Krivitski NM. Clinical measurement of blood flow in hemodialysis access fistulae and grafts by ultrasound dilution. Asaio j. 1995;41(3):M745-749.

31. Tessitore N, Bedogna V, Gammaro L, Lipari G, Poli A, Baggio E, et al. Diagnostic accuracy of ultrasound dilution access blood flow measurement in detecting stenosis and predicting thrombosis in native forearm arteriovenous fistulae for hemodialysis. American Journal of Kidney Diseases. 2003;42(2):331-341.

32. Turmel-Rodrigues LA, Bourquelot P. Prospective evaluation of failure modes in autogenous radiocephalic access for haemodialysis: good diagnostic tools are necessary for effective multimodal treatments and autogenous elbow fistulas must not be ignored. Nephrology Dialysis Transplantation. 2003;18(12):2681-2681.

33. Weitzel WF, Rubin JM, Leavey SF, Swartz RD, Dhingra RK, Messana JM. Analysis of variable flow Doppler hemodialysis access flow measurements and comparison with ultrasound dilution. American Journal of Kidney Diseases. 2001;38(5):935-940.

34. Yarar D, Cheung AK, Sakiewicz P, Lindsay RM, Paganini EP, Steuer RR, et al. Ultrafiltration method for measuring vascular access flow rates during hemodialysis. Kidney international. 1999;56(3):11291135.

35. Ciandrini A, Lodi CA, Galato R, Miscia MC, Fattori MS, Cavalcanti S. A method for monitoring vascular access function during hemodialysis. Kidney international. 2009;75(5):550-557.

36. Ashoor IF, Hughson EA, Somers MJ. Arteriovenous access monitoring with ultrasound dilution in a pediatric hemodialysis unit. Blood Purification. 2015;39(1-3):93-98. 


\section{Tables}

Table 1

Baseline demographic data.

\begin{tabular}{|ll|}
\hline Number of patients, $\mathbf{n}$ & $\mathbf{2 3}$ \\
\hline Sex male/female, $\mathbf{n}$ & $16 / 7$ \\
\hline Age(years), mean (range) & $63.45(46-80)$ \\
\hline Duration of HD (median years, range) $5.5(2-21)$ \\
Underlying disease & $22(95 \%)$ \\
- Hypertension, $\mathrm{n}(\%)$ & $13(56.5 \%)$ \\
- Coronary artery disease, $\mathrm{n}(\%)$ & $8(34 \%)$ \\
- Diabetes, $\mathrm{n}(\%)$ & \\
Caused of ESRD & $8(34 \%)$ \\
Diabetes, $\mathrm{n}(\%)$ & $7(30.4 \%)$ \\
Hypertension, $\mathrm{n}(\%)$ & $7(30.4 \%)$ \\
Glomerular diseases, $\mathrm{n}(\%)$ & $1(4.3 \%)$ \\
Other, $\mathrm{n}(\%)$ & \\
Single pool Kt/V mean $\pm \mathrm{SD}$ & $2.17 \pm 0.50$ \\
\hline \multicolumn{2}{l}{ Venous pressure (mmHg, mean $\pm \mathrm{SD}) 218 \pm 20.49$} \\
\hline
\end{tabular}

Table 2

Access flow measurement by non-invasive methods

\begin{tabular}{|c|c|c|c|}
\hline \multirow[t]{2}{*}{ Methods } & \multicolumn{3}{|c|}{ Access flow (mL/min) (median with range) } \\
\hline & Ultrasound d & $\begin{array}{l}\text { nUrea } \\
\text { dilution }\end{array}$ & $\begin{array}{l}\text { Doppler } \\
\text { ultrasound }\end{array}$ \\
\hline $\begin{array}{l}\text { Non-significant Stenosis } \\
(\leq 50 \%) \\
\text { Significant stenosis }(>50 \%\end{array}$ & $\begin{array}{l}805 \\
(748-1295) \\
625 \\
(360-850)\end{array}$ & $\begin{array}{l}828 \\
(575-1,267) \\
615 \\
(385.32-966 \\
\end{array}$ & $\begin{array}{l}604 \\
(519-799) \\
590 \\
(399-604) \\
\end{array}$ \\
\hline
\end{tabular}

Table 3. Diagnostic performance of non-invasive methods for non-significant AVF stenosis (less than 50\%).

\begin{tabular}{|l|c|c|c|c|c|}
\hline \multicolumn{1}{|c|}{ Methods } & AUC & $\begin{array}{c}\text { Sensitivity } \\
(\%)\end{array}$ & $\begin{array}{c}\text { Specificity } \\
(\%)\end{array}$ & $\begin{array}{c}\text { PPV } \\
(\%)\end{array}$ & $\begin{array}{c}\text { NPV } \\
(\%)\end{array}$ \\
\hline Ultrasound dilution & 0.55 & 73 & 40 & 78 & 33 \\
& $(95 \%$ CI, $0.26-0.85)$ & & & & \\
\hline Urea dilution & 0.63 & 73 & 80 & 92 & 57 \\
\hline Doppler ultrasound & $\begin{array}{c}0.93 \\
(95 \% \text { CI } 0.32-0.93)\end{array}$ & 80 & 100 & 100 & 80 \\
\hline
\end{tabular}

Abbreviation: AUC, Area under the ROC Curve; PPV, Positive predictive value; NPV, Negative predictive value. 
Table 4. Diagnostic performance of non-invasive methods for significant stenosis of AVF (more than 50\%).

\begin{tabular}{|l|c|c|c|c|c|}
\hline \multicolumn{1}{|c|}{ Methods } & AUC & $\begin{array}{c}\text { Sensitivity } \\
\text { (\%) }\end{array}$ & $\begin{array}{c}\text { Specificity } \\
\text { (\%) }\end{array}$ & $\begin{array}{c}\text { PPV } \\
\text { (\%) }\end{array}$ & $\begin{array}{c}\text { NPV } \\
\text { (\%) }\end{array}$ \\
\hline Ultrasound dilution & 0.74 & 89 & 46 & 57 & 83 \\
& $(95 \%$ CI,0.52-0.97) & & & & \\
\hline Urea dilution & 0.82 & 89 & 64 & 80 & 67 \\
\hline Doppler ultrasound & $\begin{array}{c}0.93 \\
(95 \% \text { CI } 0.63-1.00)\end{array}$ & 100 & 82 & 69 & 100 \\
\hline
\end{tabular}

Abbreviation: AUC, Area under the ROC Curve; PPV, Positive predictive value; NPV, Negative predictive value.

\section{Figures}

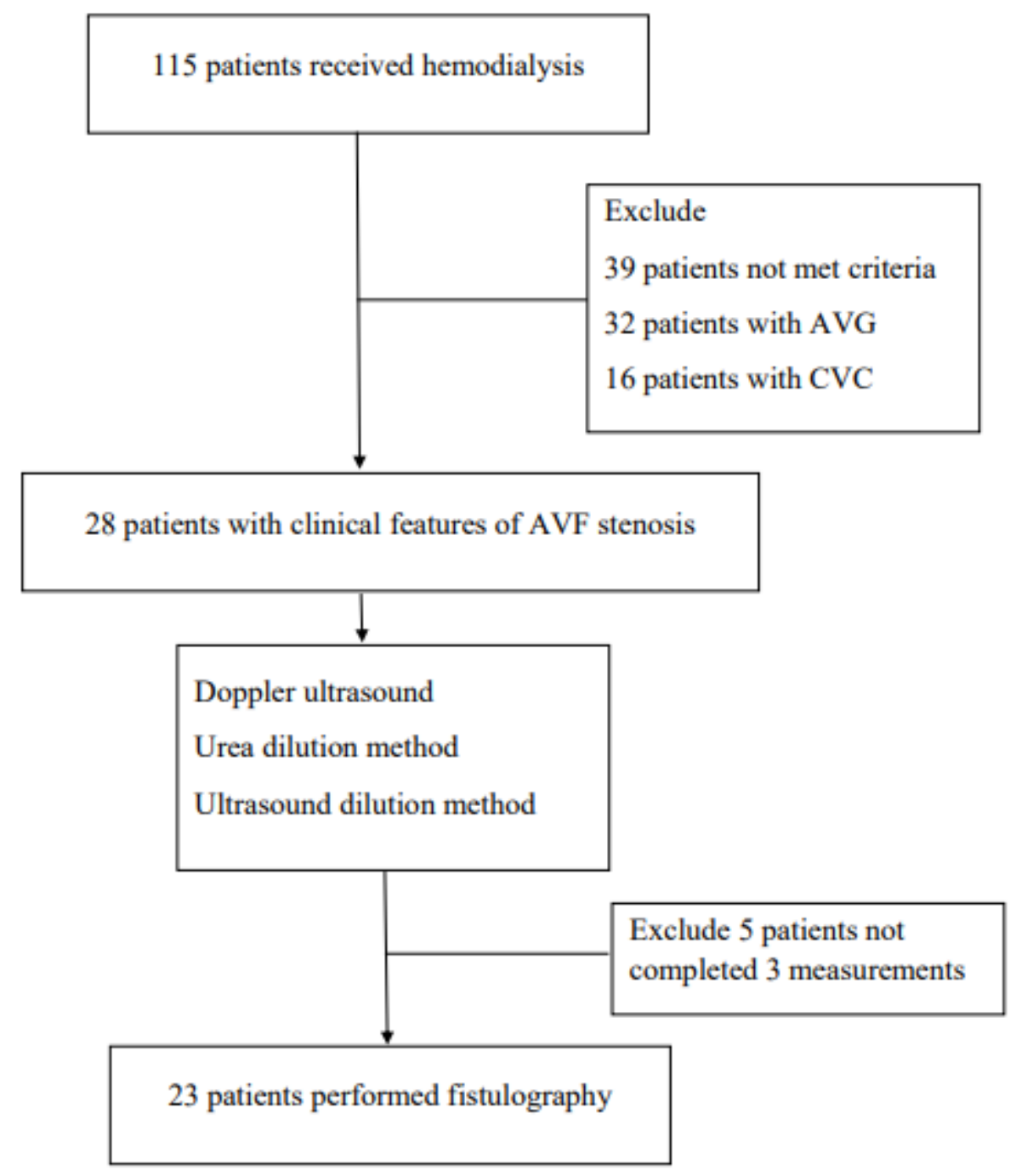


Figure 1

Flow chart of patient recruitment

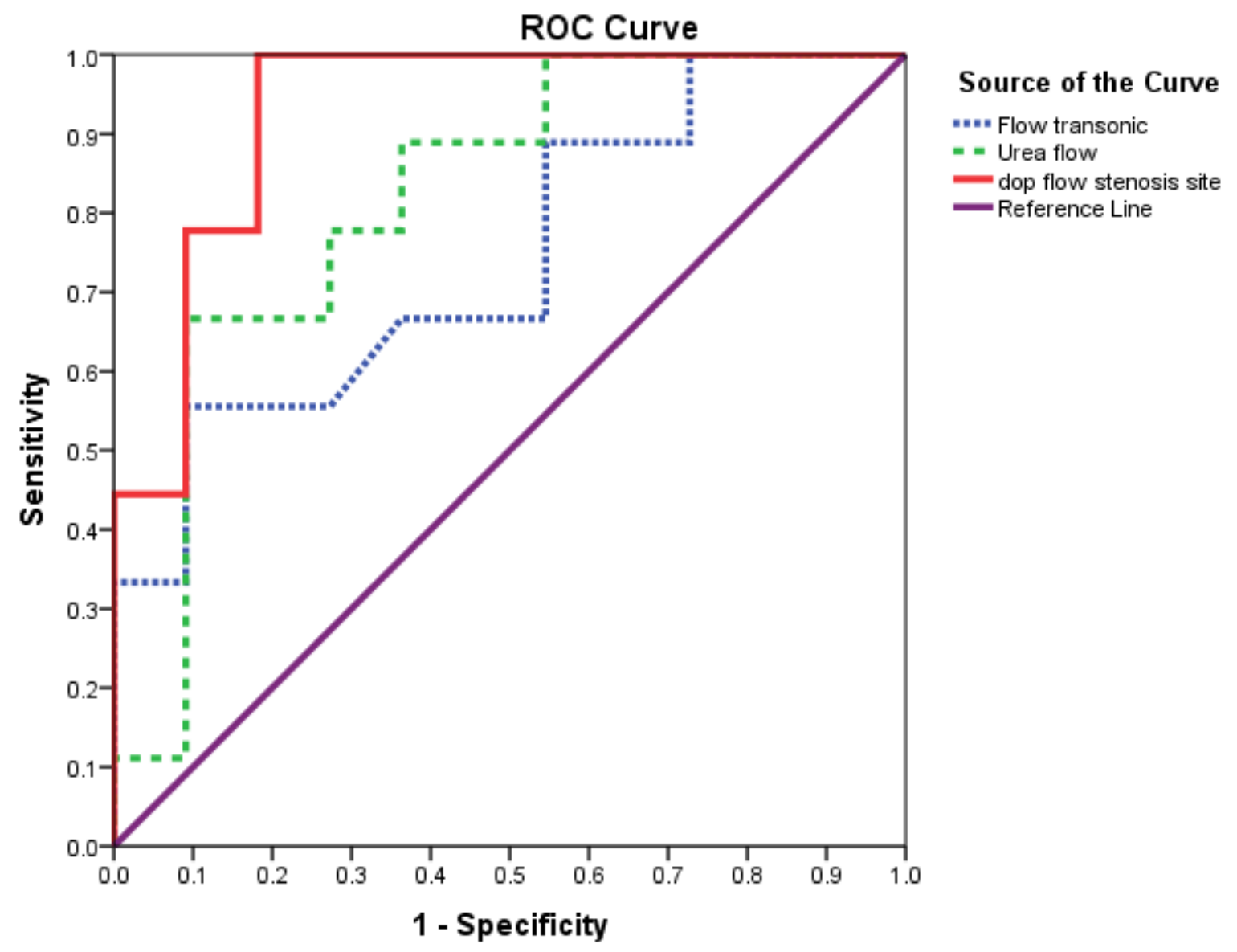

Diagonal segments are produced by ties.

Figure 2

Diagnostic performance of non-invasive methods for significant AVF stenosis 